\title{
Aspectos neuropsicológicos do transtorno obsessivo-compulsivo
}

Leonardo Fontenelle

Unidade de Transtorno Obsessivo-Compulsivo (Programa de Ansiedade e Depressão) do Instituto de Psiquiatria da Universidade Federal do Rio de Janeiro (IPUB/UFRJ). Rio de Janeiro, RJ, Brasil

\begin{abstract}
Neuropsychology is contributing to elucidate the nature of brain dysfunction associated with obsessive-compulsive disorder (OCD). Neuropsychological studies of individuals with subclinical and clinical obsessive-compulsive symptoms are reviewed here. It was observed that OCD patients may display impaired "reality monitoring”, memory for actions, non-verbal memory, visuo-spatial abilities, and executive functions. Some specific neuropsychological findings are associated with different clinical characteristics such as gender, duration of disease, severity of symptoms, and response to drug treatment. Comparative studies suggest that the neuropsychological dysfunction associated with OCD is significantly different from that seen in major depressive disorder or schizophrenia. However, many similarities were observed between patients with OCD and patients with social phobia, body dysmorphic disorder, and trichotillomania. Furthermore, studies suggest that certain neurochemical abnormalities may underlie particular neuropsychological dysfunctions found in OCD. Despite many heterogeneous results, the reviewed data seems to support the pathophysiological theory centered in the dysfunction of the fronto-striatal circuits in $O C D$.
\end{abstract}

Keywords Obsessive-compulsive disorder. Neuropsychology. Prefrontal cortex. Basal ganglia.

\section{Introdução}

A descrição de sintomas obsessivo-compulsivos associados a doenças neurológicas que acometem primariamente os gânglios da base e estudos neuroanatômicos de pacientes com transtorno obsessivo-compulsivo (TOC) têm sugerido que esse transtorno está associado a uma disfunção de estruturas frontoestriatais. ${ }^{1}$ Os estudos sobre os aspectos neuropsicológicos de indivíduos com sintomas obsessivo-compulsivos subclínicos e clínicos fornecem dados adicionais que apóiam essa hipótese.

Estudos neuropsicológicos em indivíduos com sintomas obsessivo-compulsivos subclínicos

$\mathrm{O}$ interesse nos aspectos neuropsicológicos do TOC aumentou a partir do estudo de indivíduos com sintomas subclínicos, especialmente compulsões de verificação (os chamados "verificadores" subclínicos). ${ }^{2}$ Esses indivíduos têm sido de grande utilidade para os estudos neuropsicológicos, já que apresentam sintomas semelhantes aos de pacientes com TOC e são mais freqüentes na população em geral.

\section{Memória para ações e monitorização da realidade}

Alguns estudos sobre a memória de "verificadores" subclínicos sugerem que as compulsões de verificação se devem a dificuldades em lembrar a realização de ações prévias (disfunção da memória para ações). ${ }^{2}$ Outros estudos nesses indivíduos investigaram um possível comprometimento da "monitorização da realidade", i.e. dificuldades em determinar se uma ação (p. ex. fechar o gás) foi apenas imaginada ou verdadeiramente realizada. Os resultados dessas investigações têm sido contrastantes, com relatos de comprometimento ${ }^{3}$ e de preservação de tal habilidade. ${ }^{2}$ Esses achados também são limitados, pois analisam somente um subtipo de compulsão (verificação), não podendo ser generalizados para populações clínicas que envolvem, em geral, uma diversidade de sintomas.

\section{Funções executivas}

Em outros estudos, também sugeriu-se que indivíduos com sintomas subclínicos apresentariam comprometimento das funções executivas, habilidades classicamente relacionadas com os lobos frontais. Um exemplo seria a incapacidade de mudar o cenário cognitivo, conforme observado no desempenho desses indivíduos no Wisconsin Card Sorting Test (WCST). ${ }^{4}$ Essa incapacidade já foi considerada responsável pela dificuldade de mudar ou interromper o comportamento, característica nuclear dos sintomas compulsivos. Outros testes executivos que foram realizados com dificuldade por tais indivíduos foram o Self Ordering Pointing Task (SOPT), que avalia memória de trabalho, ${ }^{5}$ e a Torre de Hanoi, que avalia a capacidade de planejamento. ${ }^{6}$

\section{Estudos neuropsicológicos em pacientes com TOC}

As principais áreas de interesse na pesquisa neuropsicológica em pacientes com TOC têm sido: (1) identificação das funções neuropsicológicas comprometidas (memória para ações, monitorização da realidade, memória não-verbal, habilidades visoespaciais, velocidade na realização dos testes e funções executivas); (2) relação entre essas disfunções e as características clínicas; (3) comparação entre as disfunções de pacientes 
com TOC e aquelas de pacientes com outros diagnósticos psiquiátricos; e (4) relação entre essas disfunções e as teorias neuroquímicas do transtorno.

\section{Memória para ações e monitorização da realidade}

Em adultos com predomínio de sintomas de verificação (TOC "verificador"), foram encontrados desempenhos tão variados quanto aqueles observados em indivíduos com sintomas subclínicos do tipo verificação. ${ }^{7-9}$ Pacientes com TOC "verificador" também demonstraram comprometimento da evocação livre de ações e da "monitorização da realidade". Entretanto, em outros estudos, notou-se que tais pacientes apresentavam preservação da "monitorização da realidade" e da memória para ações, acompanhados, porém, de menor confiança no desempenho dessas funções ${ }^{8}$ ou também da necessidade de maior vivacidade da memória para alcançar um estado de satisfação subjetiva completa. ${ }^{9}$

\section{Memória não-verbal e habilidades visoespaciais}

O comprometimento da memória não-verbal e das habilidades visoespaciais é outro achado amplamente descrito em pacientes com TOC. ${ }^{10-12}$ Classicamente, esses achados têm sustentado as teorias fisiopatológicas do TOC centradas em uma disfunção de hemisfério não-dominante. Entretanto, com base em achados recentes em pacientes com outras doenças fronto-estriatais (i.e. doenças de Huntington e de Parkinson), estudos têm sugerido que o comprometimento da memória não-verbal e das habilidades visoespaciais no TOC é resultante de uma disfunção primária dos lobos frontais. Por exemplo, no estudo de Savage et al, ${ }^{13}$ portadores de TOC foram solicitados a copiar a Figura Complexa de Rey com e sem auxílio direto da referida figura. Enquanto os indivíduos saudáveis iniciavam a cópia auxiliada da figura por seus aspectos gerais, obsessivos a iniciavam pelos detalhes. Essa estratégia de cópia foi considerada compatível com uma disfunção frontal (comprometimento da estratégia organizacional). Os que apresentavam tal comprometimento também tinham pior desempenho na cópia não-auxiliada, i.e. maior comprometimento da memória não-verbal, confirmando a hipótese de que a disfunção frontal é responsável pela aparente disfunção do hemisfério não-dominante.

\section{Lentidão}

A lentidão na realização de testes neuropsicológicos (ex: teste de Stroop e teste das Trilhas) é outro achado consistentemente descrito nesses pacientes. ${ }^{11,14,15}$ Em um estudo, a lentidão foi encontrada exclusivamente em testes que avaliam funções fronto-estriatais (SOPT e Tarefas de Aprendizado Associativo) e não em testes que avaliam a integridade de outras regiões, como as têmporo-hipocampais (Corsi's block tapping e teste de Hebb). ${ }^{15}$

\section{Funções executivas}

Assim como em indivíduos com sintomas subclínicos, os achados em adultos com TOC foram compatíveis com a presença de disfunção executiva. Em algumas investigações, foi encontrado comprometimento do desempenho em testes que, supostamente, avaliam o funcionamento das regiões órbitofrontal ou frontomedial do córtex pré-frontal, como o Teste de Alternância de Aprendizado e testes que necessitam de pensamentos ou ações rápidas e espontâneas. ${ }^{16-18}$ Em outros estudos, foi descrito desempenho insatisfatório em testes que avaliam funções relacionadas à região dorso-lateral do córtex préfrontal, como o Wisconsin Card Sorting Test (WCST) e alguns subtestes do Cambridge Neuropsychological Test Automated Battery (CANTAB), como o Spatial Working Memory e o Attentional Set-Shifting Task. ${ }^{19-22}$

Em uma revisão sobre disfunção executiva no TOC, Otto ${ }^{10}$ sugeriu que o comprometimento do desempenho em testes que avaliam funções relacionadas ao córtex pré-frontal dorso-lateral só era observado em estudos que incluíam pacientes com TOC e sintomas depressivos secundários. Entretanto, em pacientes sem sintomas depressivos, encontrava-se comprometimento do desempenho exclusivamente em testes que avaliam funções relacionadas ao córtex pré-frontal órbito-frontal, e não dorsolateral. Portanto, os achados aparentemente contrastantes quanto à localização da disfunção no córtex pré-frontal (i.e., córtex pré-frontal órbito-frontal vs. dorso-lateral) seriam resultantes de diferenças nas amostras de pacientes.

\section{Relação com características clínicas}

Em diversos estudos, já foram estabelecidas correlações positivas entre alterações neuropsicológicas específicas e aspectos clínicos. Foi encontrado pior desempenho no Matching Familiar Figures Test e no Benton Visual Retention Test em pacientes com TOC do sexo masculino, denotando maior comprometimento das habilidades visoespaciais nesse grupo. ${ }^{11}$ O maior tempo de doença se associou a pior desempenho no Objects Assembly Test, compatível com deterioração das habilidades visoespaciais, e no WCST, refletindo incapacidade de manter o cenário cognitivo. ${ }^{22}$

Pacientes com sintomas obsessivo-compulsivos mais graves também apresentaram pior desempenho na Bateria de Eficiência Mnemônica de Signoret (maior disfunção da evocação imediata visual), ${ }^{12}$ maior lentidão na realização do SOPT (pior memória de trabalho $)^{14}$ e menor número de alternâncias no Teste de Alternância de Aprendizado (maior disfunção órbito-frontal). ${ }^{16}$ A maior gravidade dos sintomas depressivos secundários também se associou à lentidão no desempenho no SOPT. ${ }^{14}$ Além disso, em um estudo não controlado, ${ }^{19} \mathrm{O}$ subgrupo de pacientes com comprometimento do desempenho no WCST (i.e., menos categorias completadas e mais erros perseverativos) foi considerado mais "psicótico", com pior prognóstico e menor inteligência. Não foram observadas correlações entre alterações neuropsicológicas e idade de início ou sintomas predominantes (lavagem, verificação ou lentidão). ${ }^{23}$

\section{Comparações com pacientes com outros diagnósticos psiquiátricos}

Pacientes com TOC apresentaram diferenças neuropsicológicas significativas em relação a pacientes com episódio depressivo maior ou com esquizofrenia. Em relação àqueles, os obsessivos apresentaram mais erros perseverativos no Objects Alternation 
Test (OAT) ${ }^{18}$ maior disfunção da memória de trabalho espacial, da velocidade da iniciação e execução motora (em subtestes do Tower of London Planning Task) e do reconhecimento espacial. ${ }^{21}$ Já quando comparados aos esquizofrênicos, tiveram mais erros perseverativos no OAT e menos erros no WCST. ${ }^{24}$

Semelhanças neuropsicológicas foram encontradas em relação a pacientes com fobia social, ${ }^{25}$ tricotilomania ${ }^{26}$ e transtorno dismórfico corporal. ${ }^{27}$ No TOC e na fobia social, foram descritos comprometimentos similares da memória não-verbal, das habilidades visoespaciais e das funções executivas. ${ }^{25}$ As semelhanças do TOC em relação à tricotilomania (comprometimento de habilidades visoespaciais $)^{26}$ e ao transtorno dismórfico corporal (disfunções mnêmica, motora e executiva $)^{27}$ são interessantes, pois convergem com modelos neurobiológicos que sugerem que esses transtornos pertencem a um espectro obsessivo-compulsivo, tendo aspectos fisiopatológicos comuns.

\section{Relação com as teorias neuroquímicas}

Um outro aspecto interessante é a relação entre alterações neuropsicológicas e anormalidades bioquímicas (serotoninérgicas, glutamatérgicas etc.) provavelmente envolvidas na fisiopatologia do TOC. Por exemplo, o comprometimento do desempenho em uma tarefa de mudança de cenário cognitivo associou-se a uma resposta embotada da prolactina ao m-CPP (meta-clorofenilpiperazina), ${ }^{28}$ sugerindo que pacientes com tal disfunção cognitiva também apresentam um tônus serotonérgico alterado. Com base nesses achados, o grupo do presente trabalho investigou a relação entre incapacidade de mudança de cenário cognitivo e reposta ao tratamento com inibidores da recaptação da serotonina.* Encontrou-se uma associação significativa entre a presença dessa disfunção e uma resposta favorável ao tratamento.

Uma outra teoria, baseada em estudos fenomenológicos e de neuroimagem funcional (ressonância magnética espectroscópica), ${ }^{29}$ sugere que o TOC estaria associado a um estado hiperglutamatérgico. Como o aumento da atividade glutamatérgica está associado à neurotoxicidade, essa teoria abre caminho para a hipótese de que tais pacientes possam apresentar sofrimento do tecido cerebral e disfunção neuropsicológica resultantes do não-tratamento ou de maior tempo de doença. Os dados de Okasha et al $^{22}$ são interessantes nesse sentido, já que apontam para uma relação entre tempo de doença e extensão do comprometimento neuropsicológico.

\section{Conclusões}

Estudos neuropsicológicos têm contribuído consideravelmente para o esclarecimento da fisiopatologia do TOC. Parte da heterogeneidade observada nos resultados pode ser resultante da diversidade sociodemográfica e clínica das amostras estudadas, bem como dos métodos de avaliação. Estudos adicionais, com amostras e investigações mais homogêneas, são necessários. Os estudos que combinam a investigação de aspectos neuropsicológicos e neuroquímicos são promissores, podendo fornecer dados adicionais sobre a fisiopatologia do transtorno.

Este trabalho foi realizado com o apoio do CNPq (Processo $\mathrm{n}^{\circ}$ 141517/00-0).

\section{Referências}

1. Saxena S, Bota RG, Brody AL. Brain-behavior relationships in obsessive-compulsive disorder. Semin Clin Neuropsychiatry 2001;6:82-101

2. Sher KJ, Frost RO, Otto R. Cognitive deficits in compulsive checkers: an exploratory study. Behav Res Ther 1983;21:357-63.

3. Rubenstein CS, Peynircioglu ZF, Chambless DL, Pigott TA. Memory in sub-clinical obsessive-compulsive checkers. Behav Res Ther 1993;31:759-65.

4. Goodwin AH, Sher KJ. Deficits in set-shifting ability in nonclinical compulsive checkers. J Psychopathol Behav Assess 1992; 14:81-92.

5. Roth RM, Baribeau J. Performance of subclinical compulsive checkers on putative tests of frontal and temporal lobe memory functions. J Nerv Ment Dis 1996;184:411-6.

6. Mataix-Cols D, Junque C, Sanchez-Turet M, Vallejo J, Verger K, Barrios M. Neuropsychological functioning in a subclinical obsessive-compulsive sample. Biol Psychiatry 1999;45:898-904.

7. Ecker W, Engelkamp J. Memory for actions in obsessive compulsive disorder. Behav Cognitive Psychother 1995;23:349-71.

8. MacDonald PA, Antony MM, Macleod CM, Richter MA. Memory and confidence in memory judgements among individuals with obsessive compulsive disorder and non-clinical controls. Behav Res Ther 1997;35:497-505.
9. Constans JI, Foa EB, Franklin ME, Mathews A. Memory for actual and imagined events in OC checkers. Behav Res Ther 1995;33:665-71.

10. Otto MW. Normal and abnormal information processing. A neuropsychological perspective on obsessive compulsive disorder. Psychiatr Clin North Am 1992;15:825-48.

11. Aronowitz BR, Hollander E, DeCaria C, Cohen L, Saoud JB, Stein $\mathrm{D}$ et al. Neuropsychology of obsessive-compulsive disorder. Neuropsychiatr Neuropsychol Behav Neurol 1994;7:81-6.

12. Dirson S, Bouvard M, Cottraux J, Martin R. Visual memory impairment in patients with obsessive-compulsive disorder: a controlled study. Psychother Psychosom 1995;63:22-31.

13. Savage CR, Baer L, Keuthen NJ. Organizational strategies and nonverbal memory in obsessive-compulsive disorder. Clin Neuropsychol 1995;9:293-4.

14. Martin A, Wiggs CL, Altemus M, Rubenstein C, Murphy DL. Working memory as assessed by subject-ordered tasks in patients with obsessive-compulsive disorder. J Clin Exp Neuropsychol 1995; 17:786-92.

15. Galderisi S, Mucci A, Catapano F, D'amato AC, Maj M. Neuropsychological slowness in obsessive-compulsive patients. Is it confined to tests involving the fronto-subcortical systems? Br J Psychiatry 1995;167:394-8.

*Fontenelle L, Marques C, Engelhardt E, Versiani M. Impaired set-shifting ability and therapeutic response in obsessive-compulsive disorder. J Neuropsychiatry Clin Neurosci 2001 (in press). 
16. Gross-Isseroff R, Sasson Y, Voet H, Hendler T, Luca-Haimovici $\mathrm{K}$, Kandel-Sussman $\mathrm{H}$ et al. Alternation learning in obsessivecompulsive disorder. Biol Psychiatry 1996;39:733-8.

17. Schmidtke K, Schorb A, Winkelmann G, Hohagen F. Cognitive frontal lobe dysfunction in obsessive-compulsive disorder. Biol Psychiatry 1998;43:666-73.

18. Cavedini P, Ferri S, Scarone S, Bellodi L. Frontal lobe dysfunction in obsessive-compulsive disorder and major depression: a clinicalneuropsychological study. Psychiatry Res 1998;78:21-8.

19. Malloy P. Frontal lobe dysfunction in obsessive-compulsive disorder. In: Perecman E. The Frontal Lobes Revisited. New Jersey: Lawrence Erlbaum Associates; 1987. p. 207-23.

20. Head D, Bolton D, Hymas N. Deficit in cognitive shifting ability in patients with obsessive-compulsive disorder. Biol Psychiatry 1989;25:929-37.

21. Abbruzzese M, Ferri S, Scarone S. Wisconsin Card Sorting Test performance in obsessive-compulsive disorder: no evidence for involvement of dorsolateral prefrontal cortex. Psychiatry Res 1995;58:37-43.

22. Okasha A, Rafaat M, Mahallawy N, El Nahas G, El Dawla AS, Sayed M, et al. Cognitive dysfunction in obsessive-compulsive disorder. Acta Psychiatr Scand 2000;101:281-5.

23. Purcell R, Maruff P, Kyrios M, Pantelis C. Neuropsychological deficits in obsessive-compulsive disorder: a comparison with unipolar depression, panic disorder, and normal controls. Arch Gen Psychiatry 1998;55:415-23.
24. Abbruzzese M, Ferri S, Scarone S. The selective breakdown of frontal functions in patients with obsessive-compulsive disorder and in patients with schizophrenia: a double dissociation experimental finding. Neuropsychologia 1997;35:907-12.

25. Cohen LJ, Hollander E, Decaria CM, Stein DJ, Simeon D, Liebowitz MR et al. Specificity of neuropsychological impairment in obsessive-compulsive disorder: a comparison with social phobic and normal control subjects. J Neuropsychiatry Clin Neurosci 1996;8:82-5.

26. Martin A, Pigott TA, Lalonde FM, Dalton I, Dubbert B, Murphy DL. Lack of evidence for Huntington's disease-like cognitive dysfunction in obsessive-compulsive disorder. Biol Psychiatry 1993;33:345-53.

27. Hanes KR. Neuropsychological performance in body dysmorphic disorder. J Int Neuropsychol Soc 1998;4:167-71.

28. Hollander E, Wong CM. The relationship between executive function impairment and serotonergic sensitivity in obsessivecompulsive disorder. Neuropsychiatry Neuropsychol Behav Neurol 1996; 9: 230-3.

29. Carlsson ML. On the role of prefrontal cortex glutamate for the antithetical phenomenology of obsessive compulsive disorder and attention deficit hyperactivity disorder. Prog Neuropsychopharmacol Biol Psychiatry 2001;25:5-26.

Correspondência: Leonardo Fontenelle

Rua Lopes Trovão, 88, apto. 1501 Bl. A - 24220-071 Niterói, RJ, Brasil

Tel.: (0xx21) 710-7857-Fax: (0xx21) 710-5161 -E-mail: Ifontenelle@bigfoot.com 